Archives

$3 \mid 1989$

Varia

\title{
Sainteté royale et sainteté dynastique au moyen âge. Traditions, métamorphoses et discontinuités
}

\section{Gàbor Klaniczay}

\section{(2) OpenEdition}

Journals

Édition électronique

URL : http://journals.openedition.org/ccrh/2932

DOI : $10.4000 /$ ccrh.2932

ISSN : $1760-7906$

Éditeur

Centre de recherches historiques - EHESS

Édition imprimée

Date de publication : 15 avril 1989

ISSN : 0990-9141

Référence électronique

Gàbor Klaniczay, « Sainteté royale et sainteté dynastique au moyen âge. Traditions, métamorphoses et discontinuités », Les Cahiers du Centre de Recherches Historiques [En ligne], $3 \mid$ 1989, mis en ligne le

13 avril 2009, consulté le 01 mai 2019. URL : http://journals.openedition.org/ccrh/2932 ; DOI :

$10.4000 /$ ccrh. 2932

Ce document a été généré automatiquement le 1 mai 2019.

Article L.111-1 du Code de la propriété intellectuelle. 


\title{
Sainteté royale et sainteté dynastique au moyen âge. Traditions, métamorphoses et discontinuités
}

\author{
Gàbor Klaniczay
}

1 En 1309, au terme d'une campagne pour l'élection de Charles Robert au trône vacant des Arpades, dynastie éteinte de Hongrie, le cardinal Gentile da Montefiore s'adressa ainsi à la noblesse hongroise : «Comme il est juste, par providence divine, le royaume de Hongrie fut dirigé par des rois catholiques, dont le premier, le saint roi Etienne et aussi quelques autres ont mérité d'être inscrits au catalogue des saints ; ils ont engendré des successeurs légitimes sous le règne desquels ce royaume a prospéré avec fertilité, a joui de la douceur de la paix, tandis que, parmi ses habitants, s'est épanouie l'unité des âmes. Mais depuis que ce royaume a été usurpé par des rois étrangers, la fertilité fut suivie par la stérilité, la douceur de la paix par le grondement de la tempête, la concorde des âmes par le désaccord détestable. Or nous, désigné par le Siège Apostolique pour réformer l'état du dit royaume, désirant offrir à tout cela un remède salutaire, convoquons en une assemblée générale les prélats et les barons et les nobles... pour qu'ils y reconnaissent communément le seigneur Charles, véritable descendant des rois saints mentionnés, en tant que roi véritable et légitime de Hongrie, leur seigneur naturel ».

2 Ces arguments, présentés par un légat pontifical italien au début du XIV siècle, peuvent servir à illustrer un phénomène de longue durée de l'histoire médiévale : la sacralisation des saints dynastiques. Ils mentionnent aussi certaines vertus attribuées au règne des rois saints : fertilité, paix, unité des âmes. Ces idées paraissent plutôt archaïsantes au début du xIve siècle : y a-t-il continuité ou résurgence ? Ces arguments présentent aussi un point de vue spécifique, qui permet de poser les problèmes historiques de la sainteté royale au Moyen Age. C'est ce que j'aimerais faire en m'appuyant sur les exemples de la Hongrie et, plus largement, de l'Europe Centrale, et en essayant de reformuler la vision d'ensemble de ce phénomène à travers une analyse comparative embrassant la longue durée du $\mathrm{x}^{\mathrm{e}} \mathrm{au}$ 
$\mathrm{xv}^{\mathrm{e}}$ siècle. Je travaille actuellement à une monographie sur ce thème, dont je souhaite présenter ici les idées essentielles ${ }^{1}$.

\section{La formation du modèle}

3 Le modèle de la sainteté royale est une création originale du Moyen Age, tirant ses éléments caractéristiques de trois traditions distinctes: des attributs sacraux et divins des souverains hellénistiques et des empereurs romains, maintenus au Moyen Age par l'Empire de Byzance; des attributs "charismatiques » des souverains germaniques et aussi d'autres souverains païens du haut Moyen Age ; enfin, bien évidemment, des formes distinctes du culte des saints, façonnées dans l'Antiquité tardive. En ce qui concerne l'évaluation historique du culte des saints rois, la question de l'interprétation des origines est liée au poids relatif qu'on accorde à ces éléments. Depuis Karl Hauck (1950), l'une des questions les plus vivement débattues est de savoir si la sainteté des rois pouvait être considérée comme une "survivance", sous une forme chrétienne, des idées « charismatiques » (germaniques ou antiques) de la royauté sacrée. La recherche détaillée sur les cultes des saints rois, reines ou princes des temps mérovingiens, due à Frantisek Graus (1965), a prouvé qu'il est très difficile de parler d'un passage direct: ces rois et reines (Sigismond, Hermenegilde, Clodovalde, Radegonde, Bathilde, Dagobert, etc.), ont accédé au titre de saint, non comme souverains "sacrés", mais plutôt parce qu'ils ont renoncé au pouvoir ou parce qu'ils en furent privés d'une façon violente, en souffrant le martyre. La sainteté ne représentait donc pas une dimension sacrale de leur pouvoir (ou de celui de leurs successeurs), et ce n'était pas une «christianisation» des attributs sacraux des anciens temps païens. On pourrait dire que les Mérovingiens n'avaient pas vraiment besoin de s'approprier les attributs du saint chrétien, parce qu'ils pouvaient garder une partie considérable de leurs attributs sacrés préchrétiens (chevelures longues, visions chamaniques, cf. saint Gontran). D'autre part, on peut comprendre la résistance du clergé mérovingien à cette transformation éventuelle de l'image du saint chrétien, qui était originellement le contrepoint du représentant du pouvoir séculier. Le saint, avec l'assistance de l'autorité divine, devait s'opposer au pouvoir séculier, en corrigeant les injustices par son propre martyre (Brown, 1981).

4 L'histoire de la formation du modèle du saint roi pose justement ce problème : comment cette opposition pouvait-elle être atténuée, quelles conditions historiques, quels contextes politiques avaient facilité cette transformation, à la suite de laquelle les fonctions royales elles-mêmes commencèrent à être interprétées comme attributs de la sainteté ? Après un bon nombre de mises au point, d'études détaillées, et de synthèses à propos des cultes des saints rois, princes et reines anglo-saxons, scandinaves, allemands, bohémiens et russes entre les $\mathrm{VII}^{\mathrm{e}}$ et $\mathrm{xI}^{\mathrm{e}}$ siècles (je ne mentionne ici que deux de ces ouvrages : Hoffmann, 1975 et Folz, 1984), je crois qu'une nouvelle vision comparative de cet ensemble peut faire avancer notre réflexion.

Le rôle origninal des royautés anglo-saxonnes reste à souligner et à expliquer : la création du modèle du roi « souffre-passion », de plus en plus assimilé dans ses traits au Christ ; la coloration de ce modèle par les vertus du roi chrétien, apôtre de son peuple, et gouvernant selon les principes chrétiens; l'utilisation de ces cultes par des prétendants d'une légitimité douteuse dans leurs luttes pour stabiliser leur trône. Les études anciennes et récentes sur le culte des saints Oswald, Edwin, Edmond, Ethelbert, Edouard le Martyr devraient permettre enfin, en s'appuyant sur les directives d'Erich Hoffmann 
(1975), de répondre à la question: pourquoi l'Angleterre fut-elle le berceau de cette nouvelle forme de culte des saints?

6 Le réseau des influences exercées par les cultes déjà formés sur ceux qui cherchent à s'établir apparaît clairement en plusieurs endroits. Néanmoins, considéré dans toute son ampleur, il peut surprendre par sa densité et sa complexité : influences ou rivalités dynastiques, ecclésiastiques, littéraires et hagiographiques, campagnes personnelles ou interventions institutionnelles; modèles anglo-saxons actifs en Norvège (saint olaf), modèles mérovingiens influençant le culte de saint Venceslas et celui des reines de l'Empire ottonien, modèle bohémien et anglo-saxon-scandinave exerçant son impact en Russie dans les cultes de saint Boris et Gleb... Il n'est pas possible de faire une exacte évaluation historique de la floraison de ces cultes d'un type nouveau dans ces territoires récemment convertis au christianisme, sans souligner le contraste remarquable entre les voies différentes de la sacralisation du souverain dans les mondes méditerranéen et carolingien (plutôt liturgique, institutionnelle et idéologique). Dans ce contexte, on pourrait qualifier le culte des saints rois comme l'une des manières propres, élaborée et empruntée à la périphérie de l'Europe chrétienne, de donner un appui surnaturel à la royauté. Ces cultes pouvaient répondre aux besoins spécifiques de ces royautés naissantes : ils aidaient à l'institutionnalisation des états chrétiens, à l'établissement des branches converties des dynasties régnantes; ils proposaient un modèle rudimentaire d'alliance entre l'Eglise et de la royauté (toutes deux en quête de stabilisation), et, finalement, comme source de leur respectabilité précaire, ils devenaient très vite un objet de rivalité parmi ces dynasties nouvelles. Pour montrer combien ce modèle diffère des voies empruntées par les souverains du « centre », il suffit de rappeler l'indifférence des Capétiens à une éventuelle canonisation de Robert le Pieux, et leur invention d'autres attributs «charismatiques » pour stabiliser leur nouvelle dynastie sur le plan du sacré (Bloch, 1983).

\section{L'achèvement du modèle - la synthèse du $\mathrm{Xl}^{\mathrm{e}}$ siècle}

7 L'exemple des cultes hongrois de saint Etienne, premier roi chrétien de la Hongrie (1000-1038), et de son fils saint Emery (Henri, mort en 1031), tous les deux instaurés en 1083 lors de la campagne pour la canonisation des cinq premiers saints hongrois, me paraît rendre compte de l'aboutissement de la formation du modèle du roi saint. Ayant pour fonction claire d'établir la légitimité du règne du roi Ladislas I (1077-1095) et de consolider l'alliance entre l'Eglise et la royauté dans un pays secoué par des soulèvements païens (1046 et 1061) et par des conflits prolongés autour de la succession, ces canonisations adoptent les armes idéologiques forgées par l'évolution pluriséculaire que je viens d'esquisser, et qui sont importées en Hongrie par Ladislas de Russie et de Bohême. En même temps, les cultes hongrois représentent une innovation importante, qui couronne la longue tendance à modérer la contradiction entre les fonctions royales et les attributs du saint chrétien : le culte de saint Etienne peut se dispenser de la gloire des martyrs. Etienne devient en effet le premier saint roi confesseur dont la façon d'exercer la souveraineté constitue la seule raison de sa sanctification.

8 L'analyse détaillée des trois légendes de saint Etienne, écrites entre 1080 et 1110, peut donner une image nuancée des cheminements historiques de l'évolution de ce modèle ; à côté des influences déjà mentionnées, on perçoit ainsi l'importance du modèle ottonien, élaboré dans les cultes des saintes Mathilde et Adélaïde (cf. Bornscheuer, 1968; Corbet, 
1986); à travers ces liens avec l'Empire, mais aussi indépendamment d'eux, on peut reconnaître l'influence du modèle clunisien français, avec l'adoption possible des éléments de l'image religieuse de Robert le Pieux; enfin, on ne peut manquer de souligner l'impact du modèle byzantin de sacralisation de la souveraineté, qui s'est enrichi, aux $\mathrm{x}^{\mathrm{e}}-\mathrm{XI}^{\mathrm{e}}$ siècles, des cultes de saints autour de Nicéphore II Phokas et Jean 1er Tzimiskès (cf. Patlagean, 1989).

En même temps, les légendes de saint Etienne illustrent la nouvelle direction de l'évolution de ces cultes: la légitimation du rôle du roi en tant que protecteur suprême des activités de l'Eglise ; en tant que garant de la paix, protecteur des pauvres, des veuves et des orphelins ; en tant qu'initiateur des nouvelles formes de piété : le culte de la Vierge (influence de Byzance), les pélerinages (influence de Cluny), etc.; en tant qu'Athleta Christi, repoussant les ennemis de la foi avec une cruauté juste ; roi législateur et savant, régnant conformément à une « raison d'État » chrétienne. Dans l'élaboration de tous ces traits, on doit souligner encore une fois le rôle politique essentiel des légendes, et il faut également élargir la documentation concernant ces cultes (comme R. Folz, 1984, le faisait à juste titre), à l'examen des chroniques, des documents législatifs et, dans le cas de saint Etienne, de ses célèbres «Instructions à son fils ».

Vers la fin $d u \mathrm{XI}^{\mathrm{e}}$ et le début du $\mathrm{XII}^{\mathrm{e}}$ siècle, le culte de saint Etienne ainsi que le développement d'autres cultes de saints rois ou princes déjà mentionnés (saints Venceslas, Olaf, Boris, Gleb), ou les nouveaux cultes qui cherchent à s'établir précisément à ce moment (saints Knut, Vladimir, Henri II, Edouard le Confesseur), ont en commun d'intégrer les modèles hagiographiques et les utilisations politiques des cultes des saints rois de la "périphérie» de l'Europe chrétienne, et les attributs de la sacralité institutionnels et politico-théologiques élaborés dans les royautés de l'Occident et dans l'Empire. Le patronat de saint Venceslas sur la Bohême, le rôle central du culte et des reliques de Saint Etienne dans le couronnement hongrois et dans la théorie politique de la « royauté apostolique » de la Hongrie, ou la position centrale des reliques, du culte et de la couronne de saint Olaf, rex perpetus Norvegiae, dans les institutions du royaume de Norvège, en illustrent bien la portée.

\section{Le saint roi chevaleresque et le saint roi mythologique}

11 Le $\mathrm{XII}^{\mathrm{e}}$ siècle représente un tournant dans l'évolution de ce modèle de sainteté, dont l'originalité, n'était guère apparente dans les recherches historiques concernant le culte des saints rois. Le modèle religieux, politique et institutionnel du culte des saints rois, dans ses formes synthétiques acquises au terme d'une évolution de cinq siècles, devient maintenant l'arme idéologique du pouvoir séculier, engagé dans une longue lutte pour la suprématie contre l'Eglise, et en particulier contre la papauté. Les canonisations royales du XII ${ }^{e}$ siècle (les saints Henri II, Edouard le Confesseur, Charlemagne, Eric) s'inscrivent dans ce contexte. A côté de l'utilisation idéologique, il est encore plus frappant de voir combien le modèle du saint roi s'est enrichi et transformé dans les nouveaux milieux de cour qui le popularisent et le développent.

12 Au siècle des croisades, dans la ligne de l'évolution déjà sensible dans les légendes des saints Olaf, Etienne et Knut, la sainteté du roi retrouve la fonction guerrière, comme Athleta Christi. Ces traits, évidemment dominants dans la légende de Charlemagne, deviennent les attributs principaux des nouveaux saints royaux : saint Eric (mort en 1160, 
canonisé en 1167), saint Ladislas (canonisé en 1192), et plusieurs princes russes, dont le plus fameux, Alexandre Newski, canonisé au XIII ${ }^{\mathrm{e}}$ siècle.

Ce nouveau modèle est soutenu par une série de chansons de gestes, histoires versifiées de plusieurs anciens et nouveaux saints rois. Le cas de Charlemagne est bien sûr évident ( cf. Paris, 1905 et Folz, 1950); on pourrait mentionner également les sagas concernant saint Olaf (qui préparent le chef d'oeuvre tardif de Snorri Sturluson) ou les histoires et légendes de saint Oswald. Un exemple très frappant est livré par les recueils de miracles, légendes et poèmes ("Vie de seint Edmund le Rei », "La Passiun de seint Edmund ») transformant la figure peu chevaleresque du roi martyr, saint Edmond, en un héros d'aventures et d'exploits militaires. D'autres cultes antérieurs, comme celui de saint Venceslas, s'enrichissent d'un nombre croissant de miracles militaires et de représentations en armure chevaleresque. En évoquant cette transformation de l'image du saint roi, on ne peut manquer de faire allusion à la vaste popularité des deux grands souverains historico-mythiques, dont la gloire chevaleresque s'ajoutait à un charisme surnaturel spécifique : Arthur et Alexandre.

Le culte de saint Ladislas reflète ces mêmes traits. Recueillant les influences des cultes des saints militaires grecs (le roi Béla III, initiateur de sa canonisation, fut élevé à Byzance), la légende de saint Ladislas le présente comme Athleta Patriae, premier candidat pour guider les croisades, et qui ne put remplir ce rôle en raison de sa mort en 1095. Dès le XIII ${ }^{\mathrm{e}}$ siècle, Ladislas est traité dans les sermons comme l'exemple parfait des vertus chevaleresques. Finalement, au XIV ${ }^{\mathrm{e}}$ siècle, pendant la floraison tardive de la chevalerie en Hongrie, saint Ladislas en devient, avec saint Georges, le patron principal; ses reliques sont même parfois conduites à la bataille pour aider les armées hongroises. Les chroniques hongroises racontent avec un nombre croissant de détails ses exploits chevaleresques, ses luttes héroïques contre les Coumans païens, dont l'un des épisodes devient une belle histoire d'aventure : il libère la fille de l'évêque de Nagyvarad ravie par un Couman, en poursuivant ce dernier à cheval; puis il le combat en duel, et après avoir réussi à le tuer avec l'aide de la femme qui avait été enlevée (et qui, selon certaines versions, semblait d'abord avoir été la complice du Couman, mais l'avait ensuite trahi), le saint roi se repose sur les genoux de celle qu'il a libérée et se laisse caresser les cheveux par elle. Les analyses iconographiques et morphologiques de cette légende non-ecclésiastique (popularisée en Hongrie du XIII ${ }^{e}$ au XV $v^{e}$ siècle par une grande série de fresques) replacent cette histoire dans le contexte mythologique des contes sur les duels initiatiques, les luttes chamaniques et les histoires d'enlèvements. On peut ici mentionner l'autre caractéristique essentielle des transformations de l'image du saint roi dès le XII siècle : la résurgence des interprétations mythologiques et folkloriques de sa figure. A côté de tels aspects figurant dans des poèmes et des chansons de gestes relatifs aux saints rois chevaleresques déjà mentionnés, relevons que la légende la plus folklorique écrite sur saint Oswald, celle de Reginald (mettant en relation la redécouverte miraculeuse de sa main droite avec un frêne, une source et un corbeau - tous trois sacrés), date du XII siècle. Les Gesta Danorum de Saxo Grammaticus, comme l'a montré Georges Dumézil (1953), empruntent la mythologie tripartie pour dépeindre les premiers rois danois. Le schéma dumézilien s'applique aussi au cas des mythes concernant le roi agriculteur, auprès des Premyslides et des Piaszt, qui se reformulent et se popularisent au XII siècle (Banaszkiewicz, 1986). Cette mythologisation du culte des saints rois, dont les éléments archaïques servaient souvent de preuves aux partisans de la continuité entre le culte 
chrétien et les idées de la royauté sacrée préchrétienne, me paraît plutôt une création originale de la civilisation du XII ${ }^{e}$ siècle.

\section{La féminisation et les légendes comme préceptes}

15 Le $\mathrm{XIII}^{\mathrm{e}}$ siècle apporte une nouvelle transformation dans le caractère et dans le profil religieux de ces cultes. Le plus frappant dans ces transformations est sans doute l'apparition d'un grand nombre de princesses saintes ou bienheureuses, qui prennent la relève des saints masculins de la dynastie royale ou princière pour sanctifier leur lignage. Ce phénomène s'observe surtout en Europe Centrale, mais il y a des parallèles en Occident et dans la Méditerranée.

La canonisation de deux reines (Marguerite d'Ecosse et Cunégonde, femme d'Henri II), s'inscrit encore dans une tradition de la sainteté des reines, qui s'enracine dans le haut Moyen Age. Les saintes Radegonde, Bathilde, Edith, Mathilde, Adélaïde). Mais la douzaine de princesses saintes ou bienheureuses qui bénéficient du succès immédiat de sainte Elisabeth de Hongrie (ou de Thuringe), canonisée quatre ans après sa mort en 1235, représente un phénomène quantitativement et qualitativement nouveau. Pour ne mentionner que les plus notables: sainte Hedvige de Silésie (1174/78-1243), la Bienheureuse Agnès de Bohême (1205-1282) et sa sœur, belle-fille d'Hedvige, sainte Anne (1203-1265), sainte Marguerite de Hongrie (1242-1270) et ses trois soeurs bienheureuses (Cunégonde, Constance et Yolande), représentent un véritable mouvement pour la sainteté au sein de ces dynasties.

17 L'analyse de la vie de ces princesses, représentées non seulement dans leurs légendes, mais aussi dans la documentation abondante de leurs procès de canonisation, et dans d'autres sources de caractère non hagiographique, à côté des traits communs de la sainteté féminine au XIII ${ }^{\mathrm{e}}$ siècle ( $c f$. Bynum, 1987 ; Vauchez, 1987), permet de relever deux thèmes qui méritent une attention particulière de notre point de vue : leur penchant à faconner leur comportement religieux en opposition symbolique à la vie de la cour royale ou princière, et leurs efforts pour réaliser les préceptes tirés des légendes d'autres saints.

En ce qui concerne le premier point, l'analyse met en évidence l'organisation de leur environnement (les monastères aristocratiques fondés pour elles ou par elles qui représentaient des métaphores vivantes de la « cour céleste » féminine, à côté des cours séculières et masculines). On peut montrer combien leurs pratiques ascétiques (jeûner, porter des vêtements austères, négliger leur corps, refuser les offres de mariages pour rester fidèles à leur époux céleste) prenaient un sens concret, si on les interprète comme un refus spectaculaire des pratiques hédonistes de la cour séculière dont elles étaient issues. Cette opposition symbolique, par le détour caractéristique de la spiritualité mendiante (qui les influençait à travers les figures puissantes des confesseurs, leurs alliés principaux), amenait ces princesses à une position inattendue de pouvoir : grâce à leur nouveau prestige, elles devenaient des médiateurs dans les conflits de famille, elles intervenaient dans les affaires politiques, juridiques, et leur charité opposait aux pratiques de la cour royale, l'idéal de la royauté selon les ordres mendiants. On ne peut manquer d'évoquer, ici, les parallèles et les différences avec le principal culte du saint roi en Occident : celui de saint Louis, (sur qui Jacques Le Goff prépare une monographie). Les confesseurs mendiants, en soutenant, en dirigeant ces cours célestes des saintes princesses en Europe Centrale, cherchaient à influencer le comportement du pouvoir 
royal dans les mêmes directions qu'en France, mais par d'autres moyens, mieux adaptés à un contexte différent.

L'autre trait à analyser dans ces cultes (et aussi dans celui de saint Louis ou de saint Louis d'Anjou, car il s'agit d'un trait général propre au XIII ${ }^{\mathrm{e}}$ siècle), est le rôle des légendes dans la formation des candidats à la sainteté. Dans les cultes d'Europe Centrale, c'est surtout l'exemple de sainte Elisabeth qui inspire l'émulation; par exemple, dans la vie de sainte Marguerite, un chapitre spécial décrit sa méditation sur les légendes de tous ses saints ancêtres (les saints Etienne, Emery, Ladislas et Elisabeth), chacun étant la source de l'un de ses mérites religieux. Voilà le principe pédagogique bien ancré dans la conscience dynastique (qui montre vers la fin du XIII ${ }^{\mathrm{e}}$ siècle, une nouvelle ascendance de l'idée du lignage saint, de la beata stirps (cf. Vauchez, 1977). L'apogée de l'histoire du culte du saint roi en Occident est atteint quand la légende, cette idéalisation postérieure, devient pour un certain nombre de rois et de princesses, un programme de vie à réaliser à la lettre, une sorte de miroir des princes.

\section{Représentation culturelle et politique dynastique}

L'impact de ces personnages du XIII ${ }^{e}$ siècle était très fort, et les dynasties du XIV siècle s'en servirent à leur manière, en utilisant les nouveaux moyens artistiques de leur temps. Ici encore, l'exemple des dynasties de l'Europe Centrale peut se révéler très instructif. A l'époque, ce sont trois nouvelles dynasties, les Luxembourg, les Angevins et les Habsbourg, qui cherchent encore une fois à renforcer leur légitimité par l'affiliation aux cultes des saints rois de la région, comme la citation du Cardinal Gentile l'a montré au début de cet article.

21 Enumérons sommairement les éléments de ces cultes : la politique de canonisation des nouveaux saints de la famille (comme celui de saint Louis d'Anjou); l'essai de faire canoniser Marguerite de Hongrie de la part des Angevins, Agnès de Bohême de la part des Luxembourg, Léopold III de celle des Habsbourg) ; le faste sans précédent des monuments funéraires, les légendiers enluminés et les représentations iconographiques des saints de la famille, qui les élevaient au statut de culte d'état; les pélerinages des familles royales à Aix, Cologne, Marburg, Rome, accompagnés d'une distribution de reliques des saints de la famille, et qui faisaient de ces rois de véritables gérants du capital de la sainteté de leurs dynasties; en patronant ces cultes, les rois voulaient de plus en plus apparaitre comme des réincarnations de leurs saints ancêtres (comme Charles IV pour Charlemagne, ou Louis d'Anjou pour saint Ladislas).

Cette propagande, fondée sur la sainteté dynastique et lignagère, banalisait en même temps l'idéal de la sacralité du souverain, incarné par ces cultes. Pour finir, il me faudra analyser les trois lignes d'évolution qui se dégagent de cette étude : la dissolution de cette forme (et d'autres formes aussi) de sacralité des rois après le xIV siècle; la formation des cultes politiques et séculiers autour de quelques souverains de la Renaissance (par exemple, Mathias Corvin), qui, malgré des différences fondamentales, montrent des similarités remarquables avec les cultes des saints rois; et finalement, le cheminement des cultes des saints rois vers une nouvelle destinée, celle de symboles nationaux. 


\section{Bibliographie}

Marc BLOCH ; Les rois thaumarturges. Étude sur le caractère surnaturel attribué à la puissance royale particulièrement en France et en Angleterre, Paris, Gallimard, 1983.

Lothar BORNSCHEUER, Miseria Regum, Berlin, Gruyter, 1968.

Peter BROWN, The cult of saints. Its rise and function in latin christianity, Chicago, University of Chicago Press, 1981.

Caroline WALKER BYNUM, Holy feast and holy fast. The religious signifiance of food to medieval women, Berkeley/Los Angeles, University of California Press, 1986.

Patrick CORBET, Les saints ottoniens. Sainteté dynastique, sainteté royale et sainteté féminine autour de l'an Mil, Sigmaringen, Thorbeke, 1986.

Robert FOLZ, Le souvenir et la légende de Charlemagne dans l'Empire médiéval, Paris, Les Belles Lettres, 1950.

Les saints rois du Moyen Age en Occident ( $\mathrm{VI}^{\mathrm{e}}$-XIII ${ }^{\mathrm{e}}$ siècle), Bruxelles, Société des Bollandistes, 1984.

Frantisek GRAUS, Volk, herrscher, heiliger im reich der Merowinger. Studen zur hagiographie der Merowingerzeit, Praga, 1965.

Karl HAUCK, « Geblütsheiligkeit », In Liber Floridus. Festschrift für Paul Lehman, St Ottilien, 1950

Erich HOFFMANN, Die heiligen kénige bei den angelsachsen und den skandinavischen vélkern. Königsheiliger un königshaus, Neumünster, Wachhloz, 1975.

Evelyne PATLAGEAN, Le Basileus assassiné et la sainteté impériale, 1989.

André VAUCHEZ, "'Beata stirps', sainteté et lignage en Occident aux XIII et XIV siècles ", in G. Duby, J. Le Goff, éd. Famille et parenté dans l'Occident médiéval, Rome, Ecole Française de Rome, 1977, pp. 397-406. Les Laïcs au Moyen Age. Pratiques et expériences religieuses, Paris, Le Cerf, 1987.

\section{NOTES}

1. J'ai eu l'occasion de présenter l'état de mes recherches aux séminaires de Jacques Le Goff et Jean-Claude Schmitt, lors de mon séjour à l'E.H.E.S.S. en janvier 1989. Pour une documentation plus détaillée sur les phénomènes mentionnés dans cet essai, et en attendant l'achèvement de la monographie, je me permets de faire référence à mes deux études déjà parues sur ce sujet: «From sacral Kingship to Self-Representation. Hungarian and European Royal Saints in the 11th and 13th Centuries", in E. Vestergaard ed., Continuity and change. A symposium. Political institutions and literary Monuments in the Middle Ages, Odense, 1986, p. 61-86. ; « Les rois saints et les Anjou de Hongrie », Alba Regia, XXII, 1985, p. 57-66. 


\section{AUTEUR}

\section{GÀBOR KLANICZAY}

Gàbor KLANICZAY est maître-assistant à l'Université de Budapest. 